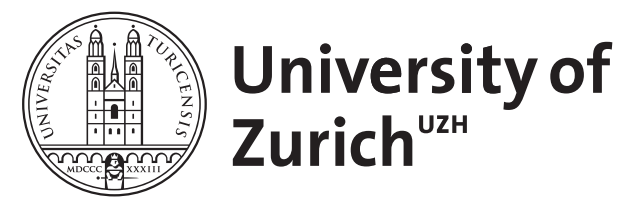

Zurich Open Repository and Archive

University of Zurich

University Library

Strickhofstrasse 39

CH-8057 Zurich

www.zora.uzh.ch

Year: 2013

\title{
Gutachterhaftung in Frankreich
}

Babusiaux, Ulrike

Posted at the Zurich Open Repository and Archive, University of Zurich ZORA URL: https://doi.org/10.5167/uzh-85387

Journal Article

Originally published at:

Babusiaux, Ulrike (2013). Gutachterhaftung in Frankreich. Kunst und Recht, 15(2):64-72. 

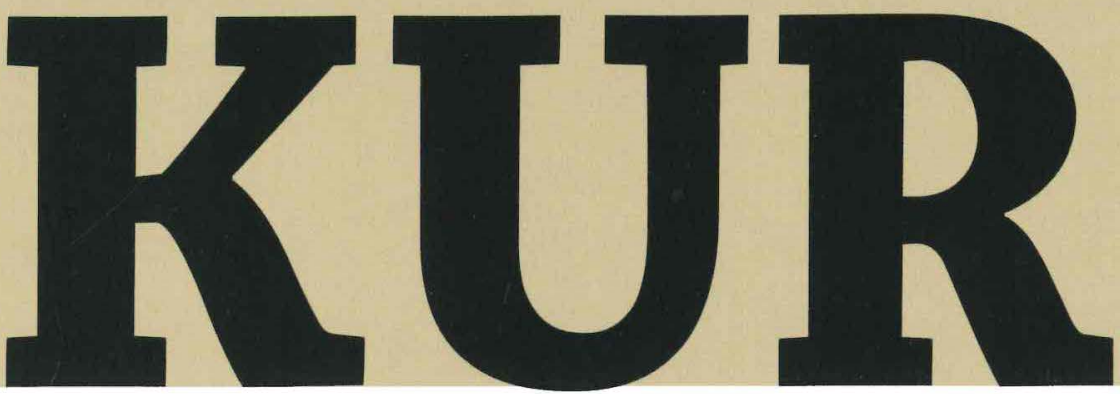

Kunst und Recht| Journal für Kunstrecht, Urheberrecht und Kulturpolitik

\section{Herausgeber: Dr. Ulf Bischof}

Aus dem Inhalt:

Wissenschaftlicher Beirat:

Dr. Michael Franz

Leiter der Koordinierungsstelle Magdeburg

\section{Robert Kirchmaier}

Leitender Regierungsdirektor bei den

Bayerischen Staatsgemäldesammlungen,

München

\section{Thomas R. Kline}

Rechtsanwalt, Andrews Kurth LLP,

Washington D.C.

Dr. Andrea F.G. Raschèr

Raschèr Consulting, Zürich

\section{Prof. Dr. Haimo Schack}

Christian-Albrechts-Universität zu Kiel

\section{Thomas Weis}

Geschäftsführer der Internationalen

Gesellschaft der Bildenden Künste, Berlin
Gestaltung des Gutachtervertrags im

Schweizer Recht

Alexander Jolles/Isabelle Roesle

Die Stellung des Gutachters im Kunsthandel und Auktionswesen

Kuno Fischer

Haftung des Kunstexperten nach deutschem Recht

Kurt Siehr

\section{Gutachterhaftung in Frankreich}

Ulrike Babusiaux 


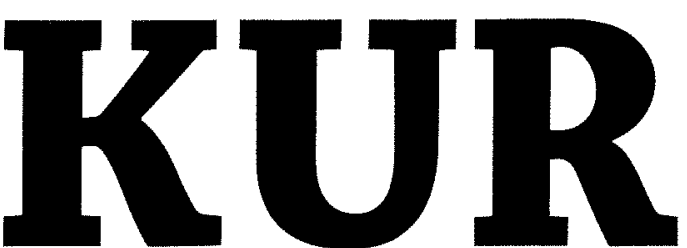

Journal für Kunstrecht, Urheberrecht und Kulturpolitik

\section{$2 \mid 2013$}

15. Jahrgang

\section{Editorial}

Liebe Leser,

die aktuelle KUR geht vor allem auf die Stellung der Experten ein. Der Kunstmarkt will verkaufen. Je besser die Ware, desto höher die Provision. Die Einlieferer qualitätsvoller Werke werden umworben. Zweifler sind weniger beliebt. Wer gibt schon gern tausende Euro für Analysen aus, um sich damit den eigenen Glauben an das Meisterwerk rauben zu lassen. Sicher haben sich Standards für Alte Meister oder zum Beispiel die Altersbestimmung chinesischer Keramik gebildet; dies gilt aber nur für ein ausgewähltes Warensegment. Mit abnehmendem Wert des offerierten Kunstwerks sinkt auch der Sorgfaltsmaßstab, und man gelangt tiefer ins Dickicht der verschiedenen Interessen der beteiligten Akteure.

Die Experten sind nicht nur an der kunsthistorischen Betrachtung interessiert, sondern naturgemäß auch an der Vergütung ihrer Leistung. Das wird dann problematisch, wenn sie selbst auf die eine oder andere Weise am Markt teilnehmen. Problematisch kann es auch sein, wenn immer wieder auf denselben Experten zurückgegriffen wird, als letzte und einzige Instanz, sei er nun Kunsthistoriker oder häufig publizierender Spezialist bzw. naher Familienangehöriger des verstorbenen Künstlers. Derart viel beschäftigt und marktbeherrschend bleibt für kritische Rückfragen wenig Zeit.

Auch die zunehmend geforderten naturwissenschaftlichen Untersuchungen sind nicht leicht $z u$ haben. Anerkannte (meist öffentliche) Institute sind rar gesät und entscheiden selbst, welche privaten Aufträge sie bei chronischem Personalmangel annehmen. Steht man nicht mit einem Dürer vor der Tür, bleibt sie bis auf Weiteres verschlossen.

Wir wollen über Experten im Kunstmarkt sprechen und hoffen, Sie damit gut zu unterhalten.

Eine informative Lektüre wünscht herzlichst Ihr

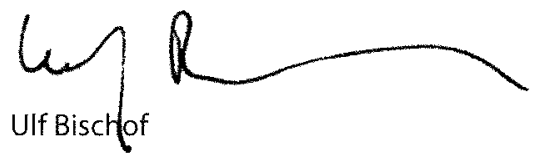

\section{Inhalt}

\section{Kunstmarkt \\ Gestaltung des Gutachtervertrags im Schweizer Recht}

Alexander Jolles/Isabelle Roesle

Die Stellung des Gutachters im Kunsthandel und Auktionswesen

Kuno Fischer

Haftung des Kunstexperten nach deutschem Recht

Kurt Siehr

„Kunst wird nicht mit den Augen gekauft, sondern mit den Ohren" oder Katalogangaben als Beschaffenheitsvereinbarung

Roland Michael Beckmann

\section{Gutachterhaftung in Frankreich}

Ulrike Babusiaux

\section{Kulturpolitik}

\section{Keine Justiz ist unfehlbar!}

Sabine Weber

\section{Rechtsprechung}

Keine Rückgabe von Antiken an die Türkei OLG Frankfurt am Main, Urteil vom 04. Februar 2013 - 16 U 161/11

Rückgabe von Raubkunst an die Republik Zypern

OLG München, Urteil vom 18. März 2013

-19 U 4878/10 


\title{
Gutachterhaftung in Frankreich
}

\author{
Ulrike Babusiaux*
}

Die französische Rechtsprechung hat die Gutachterhaftung bei den für den Kunsthandel typischen Auktionskäufen über die gesetzliche Regelung hinaus ausgeweitet. Gestützt auf ein Dekret zur Bekämpfung von Betrug im Kunsthandel von 1981 lässt sie den Gutachter (wie den Auktionator) für jede Falschangabe im Katalog haften. Die damit etablierte Garantiehaftung umfasst auch den immateriellen Schaden des „enttäuschten Kunstliebhabers". Der Gutachter haftet gesamtschuldnerisch mit dem Auktionator fünf Jahre ab Entdeckung der fehlenden Echtheit des verkauften Werkes.

\section{Kunsthandel nach französischem Modell}

- Die Verkäufe von Kunstwerken und Sammlerstücken finden in Frankreich regelmäßig als vente aux enchères publiques, also als öffentliche Versteigerungen (Auktionen), statt ${ }^{1}$. Als ventes aux enchères publiques gelten gemäß Art. L.320-2 C. com. ${ }^{2}$ Kaufverträge, die durch Zwischenschaltung eines Dritten stattfinden, der als Beauftragter des Eigentümers oder dessen Vertreters auftritt, um eine Sache anzubieten und dem Meistbietenden nach einem öffentlich zugänglichen und transparenten Versteigerungsverfahren zuzuschlagen. ${ }^{3}$ Für diese Versteigerungen gilt gemäß Art. L.321-17 C. com. grundsätzlich das allgemeine Kaufrecht ${ }^{4}$.

Da auch die sogenannten "freiwilligen Versteigerungen" bis in die jüngere Vergangenheit als staatliches Verfahren ausgestaltet waren, weist das Rechtsregime dieser Kaufverträge trotz Geltung der allgemeinen Vorschriften gewisse Besonderheiten auf, die sich auch auf die Expertenhaftung auswirken.
Prof. Dr. iur. Ulrike Babusiaux ist Inhaberin des Lehrstuhls für Römisches Recht, Privatrecht und Rechtsvergleichung der Universität Zürich

12011 galten 52\% der in Frankreich stattfindenden Versteigerungen Kunstwerken und Sammlerstücken, vgl. Conseil des Ventes, Les ventes publiques en France. Rapport d'activité 2011, 2012, S. 108f. Einsehbar unter: http://www.conseildesventes.fr/ (zuletzt eingesehen am 3.3.2013).

2 Code de commerce, zuletzt geändert am 16.2.2013. Es handelt sich um eine codification à droit constant.

3 Art. L. 320-2 Abs. I C. com.: „Constituent des ventes aux enchères publiques les ventes faisant intervenir un tiers, agissant comme mandataire du propriétaire ou de son représentant, pour proposer et adjuger un bien au mieux-disant à l'issue d'un procédé de mise en concurrence ouvert au public et transparent. (...)".

4 Art. L. 321-17 C. com.. "Les opérateurs de ventes volontaires de meubles aux enchères publiques (...) ainsi que les experts qui les assistent dans la description, la présentation et l'estimation des biens engagent leur responsabilité au cours ou à l'occasion des prisées et des ventes de meubles aux enchères publiques, conformément aux règles applicables à ces ventes."

\section{Die schrittweise Liberalisierung eines staatlichen Monopols}

Bis zum Jahre 2000 unterstand das französische Auktionswesen einem streng reglementierten staatlichen Monopol. Zurückgehend auf ein Gesetz von 1841 konnte nur derjenige als Auktionator (commissaire-priseur) tätig werden, der aufgrund des Nachweises seiner Qualifikation (Juristisches Diplom oder Kunsthistorisches Diplom nebst Prüfung und Praktikum) vom Ministerium der Justiz als solcher ernannt worden war ${ }^{5}$. Die Ernennung beinhaltete die Verpflichtung, sich der strengen disziplinarischen Aufsicht des Conseil des Ventes als Berufsverband zu unterwerfen; der Auktionator wurde zudem nach gesetzlich fixierten Tarifen bezahlt. Im Jahre 2000 ist mit Blick auf die "freiwilligen Versteigerungen" das staatliche Monopol zugunsten privatrechtlich organisierter Gesellschaften (sociétés de ventes volontaires) gefallen ${ }^{6}$. Das Jahr 2011 brachte unter dem Eindruck der europäischen Dienstleistungsrichtlinie ${ }^{7}$ eine weitergehende Liberalisierung des Auktionshandels dahin, dass sich Private und insbesondere auch EU-Ausländer beim Conseil des ventes anmelden und ohne weitere Zulassungsbedingung die Auktion durchführen können'. Die Zulassungspflicht (agrément préalable) ist damit ersatzlos gefallen; der Auktionator heißt im Gesetz nunmehr etwas sperrig "Betreiber des freiwilligen Versteigerungskaufs" (opérateur de vente volontaire) ${ }^{9}$.

5 Vgl. R. Maurice, Le commissaire-priseur et les ventes publiques de meubles, 1971.

$6 \quad$ L. $n^{\circ} .2000-642,10$ juill. 2000 portant réglementation des ventes volontaires de meubles aux enchères publiques, J.O. 11 juill., p. 10474.

7 RiL 2006/123/EG des Europäischen Parlaments und des Rates v. 12.12.2006 über Dienstleistungen im Binnenmarkt, ABl. L 376 v. 27.12.2006, S. 36-68, dazu zuletzt Duret-Robert, (Fn. 5) Rn. 10.09

8 L. n. 2011-580, 20 juill. 2011 de libéralisation des ventes volontaires de meubles aux enchères publiques, J.0. 21 juillet, p. 12441, dazu L. Mauger-Vielpeau, La réforme des enchères publiques, Droit et patrimoine 2008, S. 81-86.

$9 \quad$ Einzelheiten bei Duret-Robert (Fn. 5) Rn. 21.11-21.31. 


\section{Die Rolle des (selbständigen) Gutachters}

Die Liberalisierung des Auktionshandels hat sich auch auf die Gutachter (experts) ausgewirkt. Diese sind in Frankreich regelmäßig selbständig tätig, also nur durch einen Auftrag oder Werkvertrag mit dem Auktionshaus verbunden ${ }^{10}$. Im ursprüng lich staatlich gebundenen Auktionssystem, hatte auch der Experte eine Zulassung zu durchlaufen, die zu einer Genehmigung der Ausübung der Expertentätigkeit führte, sodass auch die Echtheit der versteigerten Kunstgegenstände nur von staatlich zugelassenen Gutachtern festgestellt werden konnte ${ }^{11}$. Diese Zulassungsbeschränkung wurde 1985 aufgegeben, 2000 wieder eingeführt und erst 2011 definitiv verworfen ${ }^{12}$. Seitdem kann sich jeder, der sich dazu berufen fühlt, als Kunstexperte betätigen.

Trotz der Veränderungen dieser Rahmenbedingungen hat sich die Rollenbeschreibung des Gutachters im Gesamtgeschehen der Auktion über alle Reformen hinweg erhalten: Nach Art. L.321-17 Abs. 1 C. com. sind Gutachter diejenigen, die dem Auktionator in der Beschreibung, Darbietung und Schätzung des Versteigerungsgutes zur Seite stehen. Aus diesem Grund - so stellt die Vorschrift klar - haften sowohl der Auktionator als auch der Gutachter während und wegen dieser Versteigerungen "nach den auf diese Kaufverträge anwendbaren Vorschriften ${ }^{\text {"13 }}$

Diese Anbindung des Gutachters an den Auktionator wirkt sich entscheidend auf Grund und Umfang der Gutachterhaftung aus: Da die Käufer eines sich als unecht erweisenden Kunstwerkes vorrangig die Rückabwicklung des Kaufes mittels der Nichtigkeitsklage (action en nullité) gegen den Auktionator anstreben, steht der aus der Unechtheit des Kunstwerkes erwachsene Schadenersatzanspruch gegen Auktionator und Gutachter im Schatten dieser Klage ${ }^{14}$.

Zum besseren Verständnis der den Gutachter nach französischem Recht treffenden Haftung ist es daher notwendig, zunächst die Nichtigkeitsklage des Käufers gegen den Auktionator zu betrachten, bevor ein Blick auf die gesetzlichen Regeln

10 Zu dieser Tendenz vgl. S. Lequette-de Kervenoaël, Lauthenticité des oeuvres d'art, 2006, Rn. 487, S. 433; zuletzt Duret-Robert, (Fn. 5) Rn. 141.12. Ein historischer Abriss bei E. Turquin, in: A. Baron et al. (Hg.), Marché de l'Art, l'expert dans tous ses états, 2004, S. 9-12. Die Haftung von angestellten Gutachtern bleibt daher hier außer Betracht.

11 Zur Kritik vgl. Mauger-Vielpeau (Fn. 8) S. 85.

12 Einzelheiten zur Entwicklung bei Lequette-de Kervenoaël (Fn. 10) Rn. 488, S. 434.

13 Art. L. 321-17 Abs. 1 C. com.: "Les opérateurs de ventes volontaires de meubles aux enchères publiques mentionnés à l'article L. 321-4 et les officiers publics ou ministériels compétents pour procéder aux ventes judiciaires et volontaires ainsi que les experts qui les assistent dans la description, la présentation et l'estimation des biens engagent leur responsabilité au cours ou à l'occasion des prisées et des ventes de meubles aux enchères publiques, conformément aux règles applicables à ces ventes."

14 Zum Vorfragecharakter der Nichtigkeitsklage vgl. Duret-Robert (Fn. 5) Rn. 144.31-144.32, Rn. 144.41-144.44. der vertraglichen wie der deliktischen Haftung zu werfen ist, die grundsätzlich auch auf den Kunstexperten Anwendung finden. Im Zentrum wird sodann das Sonderrecht stehen, das die Rechtsprechung mit Blick auf die Haftung des Gutachters im Kunsthandel geschaffen hat.

\section{Rahmenbedingungen der Gutachterhaf- tung}

Die berühmtesten Kunstmarktfälle des französischen Rechts (affaire Poussin ${ }^{15}$ ) kreisen um die Frage, unter welchen Voraussetzungen der Käufer (oder gar der Verkäufer) berechtigt ist, die Nichtigkeit des Vertrages wegen Irrtums geltend zu machen, wenn sich das als "echt" gekaufte Werk als nicht authentisch erweist $^{\mathbf{1 6}}$. Dabei haben die Regeln der Irrtumsanfechtung die an sich für die Sachmängel vorgesehene Haftung verdrängt. Dies ist nicht nur der Tatsache geschuldet, dass bis 2005 die Sachmängelgewährleistung beim Kauf nur im Rahmen der kurzen Frist (bref délai) erhoben werden konnte ${ }^{17}$. Vielmehr wird die Garantie für versteckte Mängel (garantie pour vices cachés) auch theoretisch als nicht einschlägig bei EchtheitsmängeIn eines Kunstwerkes angesehen ${ }^{18}$, vorrangig deshalb, weil man argumentiert, die Frage der Echtheit sei eine Frage der Willensbildung, nicht ein Problem der schlechten Qualität der Kaufsache ${ }^{19}$.

\section{Die Nichtigkeitsklage wegen Irrtums über die Echtheit des Kunstwerkes}

Nach den allgemeinen Regeln des französischen Irrtumsrechts muss der Irrende beweisen, dass die Fehlvorstellung eine wesentliche Eigenschaft der Sache betraf, und dass dieser Irrtum entscheidend für den Kaufkonsens war ${ }^{20}$. Weiter darf dem Käu-

15 Berühmt die aff. Poussin, Cass. civ. 1re, 22 fév. 1978, Bull. civ. $n^{\circ} 74$. Weitere Fälle: Cass. civ. 1re, 30 sept. 2008, n06-20.298, 06-21.254, Bull. civ. I n 217 „Dali“; Cass. civ. 1 re, 27 fév. 2007, n” 02-13.420, 03-21.179, Bull. civ. n 90, D. 2007, 1632 note Gauthier "Sésostris III". Alle Entscheidungen finden sich im Volltext auf: http://www.legifrance.gouv.fr/ (Menupunkt: Jurisprudence judiciaire; zuletzt eingesehen am 3.3.2013).

16 Ein Überblick dazu bei J. Ghestin, L'authenticité, l'erreur et le doute, in: Etudes Catala, 2001, S. 457-468; Zum praktischen Uberwiegen der Nichtigkeitsklage vgl. auch F. Duret-Robert, Ventes publiques: les garanties dont bénéficient les acheteurs, Droit et Patrimoine 2008, S. 52

172005 erfolgte die Anpassung auf eine zweijährige Frist vgl. Art. 1648 C. civ.: "L'action résultant des vices rédhibitoires doit être intentée par l'acquéreur dans un délai de deux ans à compter de la découverte du vice."

18 Einzelheiten dazu bei F. Labarthe, La valeur contractuelle du catalogue dans les ventes volontaires de meubles aux enchères publiques, Rec. Dalloz 2011, 1779, S. 18-20.

19 Zum Verhältnis von erreur und vices cachés vgl. Cass. civ. Ire, 28 juin 1988, D. 1988, 450 sowie Cass. civ. 3e, 7 juin 2000, D. 2002 Sormm. 1002, dazu H. Capitant/F. Terré/Y. Lequette, Les grands arrêts de la jurisprudence civile, t. 2, 122008, Rn. 267-268, S. 719-727; mit Blick auf den Kunsthandel vg1. Duret-Robert (Fn. 5) Rn. III.09

20 Zum erreur vgl. Cass. civ. Ire, 22 fév. 1978, D. 1978, 601 sowie Cass. civ. 1re, 13 déc. 1983, D. 1984, 340, dazu Capitant/Terré/ Lequette (Fn. 19) Rn. 147-148, S. 30-54; zu den Voraussetzungen 
fer kein unentschuldbarer Irrtum (erreur inexcusable) zur Last gelegt werden. Diese Voraussetzungen liegen bei Fehlkäufen auf dem Kunstmarkt regelmäßig vor: Zum einen ist die Echtheit des Werkes gerade bei Kunstwerken eine wesentliche Eigenschaft, zum andern wird sie auch regelmäßig entscheidungserheblich für den Abschluss des Kaufes sein. Drittens wird man dem Kunstliebhaber den Irrtum nachsehen können, wenn sogar Experten irren oder geirrt haben ${ }^{21}$. Ebenso großzügig ist der zeitliche Rahmen für die Erhebung der Nichtigkeitsklage: Art. $1304 \mathrm{C}$. civ. bestimmt, dass die Nichtigkeitsklage binnen 5 Jahren nach Entdeckung des Irrtums verjährt. Solange die fehlende Echtheit mithin unentdeckt bleibt, tritt auch keine Verjährung ein. Absolute Grenze ist - und dies erst seit 2008 - die Ausschlussfrist (délai butoire) von zwanzig Jahren (art. 2232 C. civ) ${ }^{22}$. Auf diese Weise kann der enttäuschte Käufer auch noch Jahre nach der Versteigerung eine Nichtigkeitsklage gegen das Auktionshaus erheben, um die Rückabwicklung des Kaufes zu erreichen, also seinen Kaufpreis nebst Zinsen zurückzuerhaiten und das im Ergebnis wertlose oder zumindest im Wert stark hinter den Vorstellungen zurückbleibende Bild zurückzugeben.

Für den Gutachter ist der Ausgang dieses Verfahrens insoweit von Bedeutung, als sich die Höhe seiner Haftung danach richtet, ob der Käufer mit der Nichtigkeitsklage durchgedrungen ist oder nicht. Entscheidend ist dabei, ob die Nichtigkeitsklage durch eigenes Verschulden des Käufers scheitert oder an Umständen, die der Käufer nicht zu vertreten hat. Beruht das Scheitern der Nichtigkeitsklage auf Verschulden des Käufers, der zum Beispiel die Verjährungsfrist hat verstreichen lassen, wird der Gutachter nicht voll in die Haftung genommen. Dagegen lassen sich Entscheidungen finden, die dem Gutachter das Insolvenzrisiko des Verkäufers aufbürden, das heißt den Schadenersatz des Käufers damit begründen, dass der an sich rückgabepflichtige Verkäufer insolvent oder unauffindbar verschwunden ist ${ }^{23}$.

\section{Die Gutachterhaftung als Anwendungsfall der allgemeinen Vorschriften}

Die Gutachterhaftung richtet sich grundsätzlich nach den allgemeinen Vorschriften des Vertrags- bzw. des Deliktsrechts. Dass dies auch für Begutachtungen im Kunsthandel von Bedeutung ist, folgt schon aus der Regelung des Auktionskaufes in Art. 321-17 Abs. 1 C. com., der allgemein auf die Regeln verweist, die auf diese Kaufverträge Anwendung finden ("conformément aux règles applicables à ces ventes"). Da die Versteigerung als

im Kunsthandel vgl. Duret-Robert (Fn. 5) Rn. 91.11-91.24.

21 Der großzügige Maßstab etwa in Cass. civ. 1 re, 31 mai 2007, $n^{\circ}$ 05-17.203 (non publié); Einzelheiten zum erreur excusable zuletzt bei Duret-Robert (Fn. 5) Rn. 101.41-101.46.

22 Art. 2232 C. civ.: "Le report du point de départ, la suspension ou l'interruption de la prescription ne peut avoir pour effet de porter le délai de la prescription extinctive au-delà de vingt ans à compter du jour de la naissance du droit." Einzelheiten zur Verjährung aus Sicht des Kunsthandels bei Duret-Robert (Fn. 5) Rn. 93.11-93.21. Vgl. Lequette-de Kervenoaël (Fn. 10) Rn. 548-1, S. 487 f.
Kaufvertrag (vente) im Sinne des Code civil anzusehen ist, sind mithin die Regelungen des allgemeinen Schuldrechts und des Deliktsrechts auch für die Expertenhaftung heranzuziehen ${ }^{24}$. Dabei ist zu beachten, dass beide Haftungsregime nach der Rechtsprechung der Cour de cassation nicht kumuliert werden können, sondern streng alternativ zur Anwendung kommen (principe du non-cumul' ${ }^{25}$ ).

\section{a. Die Vertragshaftung (Art. 1147 C. civ.)}

Die Vertragshaftung ist gemäß Art. 1147 C. civ. eine Nichterfüllungshaftung, wobei den Schuldner nur die höhere Gewalt entlastet (Art. 1148 C. civ.). Bei der Feststellung, ob überhaupt eine Nichterfüllung vorliegt, kommt die grundlegende Unterscheidung von Werk- und Dienstpflicht zum Tragen ${ }^{26}$ : Schuldet der Schuldner die Erreichung eines Erfolges (obligation de résultat, Werkpflicht), führt Art. 1147 C. civ. schon bei dessen Nichteintritt zur Haftung und der Schuldner kann sich nur durch Hinweis auf eine äußere Ursache, insbesondere höhere Gewalt, entlasten; trifft ihn dagegen nur eine Dienstpflicht (obligation de moyens), muss der Gläubiger darlegen, dass der Schuldner sich nicht wie ein bon père de famille verhalten hat, also die Sorgfaltspflichten verletzt hat, die eine entsprechende objektiv zu bestimmende Vergleichsperson in der Situation des Schuldners eingehalten hätte ${ }^{27}$. Auf den Gutachter gemünzt bedeutet dies, dass er sich an die Begutachtungsstandards zu halten hat, insbesondere auf die üblichen Hilfsmittel für die Ermittlung der Echtheit des Werkes zurückgreifen muss. ${ }^{28}$ Aufgrund der Unwägbarkeiten, die mit Abgabe eines Gutachtens verbunden sind, gilt - unabhängig von der typologischen Einordnung des Gutachtenvertrages - für den Experten grundsätzlich eine bloße Dienstpflicht, das heißt eine obligation de moyens ${ }^{29}$. Die Rechtsprechung hat die der Dienstpflicht innewohnenden Beschränkungen zunächst recht genau respektiert. So hat z.B. die Cour d'appel von Bordeaux einen Gutachter nicht haften lassen, weil die fehlende Echtheit eines Möbelstücks nur bei der Demontage erkennbar

24 Die Auktionskäufe bleiben dem Zivilrecht zugeordnet und sind keine Handelsgeschäfte. Zu entsprechenden Diskussionen bei der Reform von 2011, vgl. Duret-Robert (Fn. 5) Rn. $11.41 \mathrm{mwN}$. Zur Geltung der allgemeinen Regeln vgl. ders., aaO., Rn. 142.11.

25 Grundlegend Cass. civ., 11 janv. 1922, DP 1922, I, 16; S. 1924, 1, 105: „(...) Mais attendu que c'est seulement en matière de délit ou de quasi-délit que toute faute quelconque oblige son auteur à réparer le dommage provenant de son fait; que les articles 1382 et suivants sont sans application lorsqu'il s'agit d'une faute commise dans l'exécution d'une obligation résultant d'un contrat (...)", dazu Capitant/Terré/ Lequette (Fn. 19) Rn. 181, S. 279-285 mwN.

26 Vgl. G. Viney/P. Jourdain, Les conditions de la responsabilité, in: J. Ghestin, Traité de droit civil, 32006, Rn. 522-555, S. 506-557; zuletzt Ph. Le Tourneau, in: ders. (Hg.), Droit de la responsabilité et des contrats, Dalloz Action 2012/2013, 92012, Rn. 3209-3242.

27 Le Tourneau (Fn. 26) Rn. 3216.

28 Für Einzelheiten (und Rechtsprechung) vgl. Lequette-de Kervenoaêl (Fn. 10) Rn.523, S. 462 sowie Duret-Robert (Fn. 5) Rn. 142.33 mit einer Liste Rn. 62.22

29 Für Einzelheiten vgl. Lequette-de Kervenoaël (Fn. 10) Rn. 522, S. 461 . 
gewesen wäre ${ }^{30}$. Die Demontage war aber weder vereinbart noch den üblichen Standards entsprechend. Ebenso wenig erklärte die Cour d'appel von Paris einen Experten für schadenersatzpflichtig, der keine Röntgenaufnahmen vorgenommen hatte, weil diese Art von Untersuchung für den fraglichen Typus von Kunstwerk unüblich gewesen sei und gesondert hätte vereinbart werden müssen ${ }^{31}$. Damit einher geht die Begrenzung der Haftung auf die Standards und den Kenntnisstand im Moment der Begutachtung; abweichende wissenschaftliche Erkenntnisse, die nach dem Erstellen des Gutachtens hinzugetreten sind und nicht vorhersehbar waren, können naturgemäß keine Haftung begründen, wenn der Gutachter sie nicht beachtet hat ${ }^{32}$

\section{b. Die Deliktshaftung (Art. 1383 C. civ.)}

Ähnliches gilt auf der Ebene der Deliktshaftung. Hier kommt nur eine Haftung für négligence, also die sogenannte quasideliktische Haftung ${ }^{33}$ nach Art. 1383 C. civ. in Betracht ${ }^{34}$, da der Gutachter regelmäßig nicht mit der Intention tätig werden wird, den Käufer zu schädigen ${ }^{35}$. Maßgeblich ist also die Feststellung eines Verhaltensfehlers, der ohne bösartige Gesinnung zustande gekommen ist und der durch größere Aufmerksamkeit hätte verhindert werden können ${ }^{36}$. Auch hier kommt es auf eine objektive Würdigung des Verhaltens und auf eine Umschreibung der Sorgfaltspflicht aus der Perspektive eines sorgfältigen bon père de famille $\mathrm{an}^{37}$. In dieser Umschreibung lag bis vor kurzem eine Schwierigkeit der quasi-deliktischen Haftung, denn es bedurfte der besonderen Begründung, dass der Gutachter die Sorgfaltspflicht gegenüber dem Käufer verletzt habe, zu dem er keine vertragliche Verbindung aufwies.

Mit einer Entscheidung von 2006 hat die Cour de cassation diese Schwierigkeiten überwunden und die Schleusen der deliktischen Haftung bei der Verletzung von Drittinteressen weit geöffnet ${ }^{38}$. Die Assemblée Plénière entschied, dass die deliktische Sorgfaltspflichtverletzung gerade auch aus der Verletzung eines Vertrages mit einem anderen hergeleitet werden kann:

30 C. A. Bordeaux, 28 sept. 2009, dazu F. Kendérian, Expertise de mobiliers et d'oeuvres d'art: quand l'obligation de moyens de l'expert vient source d'irresponsabilité civile, Rec. Dalloz 2010, 749. Im Fall ging es um die vertragliche Haftung des Experten, insbesondere die Weigerung des Auftraggebers, die Expertise zu bezahlen.

31 Vgl. Duret-Robert (Fn. 16) S. 57.

32 Beispiele dazu bei Duret-Robert (Fn. 16) S. 58.

33 Vgl. Art. $1370 \mathrm{C}$. civ.: "Certains engagements se forment sans qu'il intervienne aucune convention, ni de la part de celui qui s'oblige, ni de la part de celui envers lequel il est obligé. (...) Les engagements qui naissent d'un fait personnel à celui qui se trouve obligé, résultent ou des quasi-contrats, ou des délits ou quasi-délits ; ils font la matière du présent titre."

34 J. Julien, in: Le Tourneau (Fn. 26) Rn. 6704.

35 Duret-Robert (Fn. 16) S. 57.

36 Julien (Fn. 34) Rn. 6705.

37 Zu den Grundlagen dieser Theorie vgl. Julien (Fn. 34) Rn. 6706; Viney/Jourdain (Fn. 26) Rn. 462-482, S. 400-428.

38 Cass. ass. plén., 6 oct. 2006, ñ 05-13.255, D. 2006, p. 2825: „(..)
„Der Vertragsdritte kann sich im Rahmen der deliktischen Haftung, auf einen Versto $B$ gegen den Vertrag berufen, wenn ihm dadurch ein Schaden entstanden ist. ${ }^{\text {“39 }}$

Für die Frage der Gutachterhaftung bedeutet dies nichts anderes, als dass der Käufer seinen auf Art. 1383 C. civ. gestützten Anspruch damit begründen kann, dass der Gutachter im Rahmen seines Begutachtungsvertrages mit dem Auktionator die gültigen gutachterlichen Standards nicht eingehalten hat. ${ }^{40}$ Die - an sich notwendige - Drittbezogenheit dieser Verpflichtung wird dabei mehr vorausgesetzt als weiter geprüft. Immerhin gilt auch hier die Begrenzung der Dienstpflicht auf die tatsächlich erreichbaren Kenntnisse zum Zeitpunkt der Gutachtenerteilung. Entspricht der Irrtum des Gutachters über die Echtheit des Kunstwerkes zum Zeitpunkt der Gutachtenerstellung dem state of the art, ist kein Sorgfaltsverstoß feststellbar.

\section{Die richterrechtliche Statuierung einer Garantiehaftung}

Die richterliche Praxis mit Blick auf den Kunstmarkt sieht freilich vollkommen anders aus als dies die skizzierte gesetzliche Regelung nahelegt. Nach Meinung der Rechtsprechung trifft den Gutachter eine Garantiehaftung, die mithin auch eingreift, wenn dem Gutachter kein Sorgfaltspflichtverstoß in vertraglicher oder deliktischer Hinsicht nachzuweisen ist ${ }^{41}$.

Diese Rechtsprechung ist zunächst näher zu betrachten, sodann auf ihre Begründung zu prüfen und dogmatisch einzuordnen.

\section{Zwei Grundsatzentscheidungen der Cour de cas- sation}

Zwei Entscheidungen der Cour de cassation werden immer wieder als grundlegend für die Abkehr vom Grundsatz der Dienstpflicht in der Rechtsprechung zitiert. Die erste stammt aus dem Jahre 1995 und betraf ein Bild des Künstlers Auguste Herbin ${ }^{42}$, die zweite, aus dem Jahre 2007, handelte von einem Gemälde des Malers Jean Dufy ${ }^{43}$.

Mais attendu qu'un tiers à un contrat peut invoquer, sur le fondement de la responsabilité délictuelle, un manquement contractuel dès lors que ce manquement lui a causé un dommage (...)" dazu $G$. Viney, Introduction à la responsabilité, in: Ghestin (Fn, 26) 32007, Rn. 215-3-215-4, S. 605-611; Capitant/Terré/Lequette (Fn. 19) Rn. 177, S. 244-255 mwN; mit Blick auf den Kunsthandel vgl. DuretRobert (Fn. 5) Rn. 142.35.

39 Cass. ass. plén., 6 oct. 2006, n05-13.255.

40 Zur Kritik vgl. P. Ancel, Retour sur l'arrêt de l'Assemblée plénière du 6 octobre, à la lumière du droit comparé, in: M. Fabre-Magnan et al. (Hg.), Etudes Viney, 2008, S. 23-37.

41 Zu diesem Widerspruch vgl. Duret-Robert (Fn. 16) S. 57.

42 Cass. civ. 1re, 7 nov. 1995, $n^{\circ}$ 93-11.418, Bull. civ, $n^{\circ} 401$.

43 Cass. civ. 1re, 3 avx. 2007, $n^{\circ} 05-12.238$, Bull. civ. $n^{\circ} 141$, dazu 1. Gallmeister, Responsabilité des experts en matière de vente d'oeuvres d'art, Rec. Dalloz 2007, 2228 sowie F. Baillet Bouin, Le commissaire-priseur, responsable de plein droit envers l'adjudicataire d'une cuvre d'art? Rec. Dalloz 2007, 2288-2292. 


\section{a. Entscheidung „Herbin“ (1995)}

In der Sache "Herbin" entschied das Gericht, der Gutachter, der das Bild dem genannten Herbin zugeschrieben hatte, hafte für den später aufgedeckten Irrtum, obgleich der gerichtliche Sachverständige kein Fehlverhalten des Gutachters feststellen konnte ${ }^{44}$ :

„Unter Beachtung von Artikel 1147 des Code civil: Unter der Erwägung, dass das Urteil, um Frau $Y$ mit ihrer Haftungsklage gegen Herrn $Z$ abzuweisen, der ihr ein Echtheitszertifikat erstellt habe, festhält, dass die gerichtlichen Sachverständigen, welche anschließend zur Annahme der Echtheit gelangt seien, zu diesem Schluss nur durch Untersuchungen gelangt seien, welche sie selbst als, anspruchsvoll und schwierig bezeichnet hätten, so dass gegenüber Herrn Z keinerlei Fehlverhalten festgestellt werden' könne, weil er ,sich von dem äußeren Erscheinen des Bildes habe täuschen lassen und seine Meinung ohne die Vornahme einer derart gründiichen Untersuchung geäußert habe, und zwar aufgrund seines Eindrucks als Kunstexperte'. Unter der Erwägung, dass die Cour d'appel derart entschieden hat, obwohl der Gutachter, der die Echtheit eines Kunstwerkes behauptet, ohne seine Äußerung mit Vorbehalten zu versehen, seine Haftung durch diese Behauptung auslöst, hat [das Gericht] den oben genannten Gesetzestext verletzt."

Hauptargument der Cour de cassation war mithin, dass das Gutachten keine Vorbehalte enthalte und die Echtheit ohne Einschränkung behaupte, weshalb der Gutachter für seine Echtheitsbehauptung einzustehen habe, obwohl die fehlende Echtheit zum Zeitpunkt der Begutachtung kaum feststellbar war.

\section{b. Entscheidung „Dufy“ (2007)}

Die Entscheidung "Dufy" von 2007 betraf die deliktische Haftung im Verhältnis von Gutachter und Käufer: Hier hatte der Gutachter das Bild, das am 22. November 1989 versteigert wurde, dem Maler Jean Dufy zugewiesen. Wie das gerichtliche Gutachten ausführte, gab es zu diesem Zeitpunkt keinerlei Grund, an der Authentizität des Werkes zu zweifeln. Erst im Jahre 2001, also mehr als 10 Jahre später, kam ein anderer Gutachter aufgrund neuen Materials und neuer wissenschaftlicher Erkenntnisse zu dem Ergebnis, es liege eine Fälschung vor. Nach den Grundsät-

44 Cassciv. 1re, 7 nov. 1995, n 93-11.418, Bull. civ. $n^{\circ} 401$ : „Vu l'article 1147 du Code civil; Attendu que pour débouter Mme Y... de son action en responsabilité contre $M$. $Z$..., qui lui avait délivré un certificat d'authenticité de l'ceuvre, l'arrêt énonce que les experts judiciaires qui avaient ensuite conclu à l'authenticité n'étaient parvenus à cette conclusion qu'après des opérations qu'ils avaient eux-mêmes qualifiées de "délicates et difficiles", et qu'aucune faute ne pouvait être retenue contre $M . Z$... pour "avoir été abusé par l'apparence du tableau et avoir donné son avis sans recourir à un examen aussi minutieux, mais en fonction de son impression de spécialiste en peinture; Attendu qu'en se déterminant ainsi, alors que l'expert qui affirme l'authenticité d'une oeuvre d'art sans assortir son avis de réserves engage sa responsabilité sur cette affrmation, la cour d'appel a violéle texte susvisé; (...) ; dazu Lequette-de Kervenoaël (Fn. 10) Rn. 534 , S. 475 mwN. zen der Dienstpflicht (Vertrag) wie der négligence (Delikt) hätte man den Gutachter nicht verurteilen können, da es zum Zeitpunkt der Begutachtung auch dem objektiv sorgfältigen Gutachter nicht möglich gewesen wäre, die Fälschung zu erkennen. Die Cour de cassation hielt demgegenüber lapidar fest ${ }^{45}$ :

"Aber in Erwägung dessen, dass die Cour d'appel, die zutreffend festgehalten hat, dass gegenüber dem Opfer des Irrtums der Auktionator oder der Experte, der die Echtheit eines Kunstwerkes behauptet hat, ohne seine Äußerung mit Vorbehalten $z u$ versehen, seine Haftung aufgrund dieser Behauptung begründet (...)."

Damit schloss das Gericht den Einwand des Gutachters, dass ihm eine Erkenntnis der Fälschung gar nicht möglich gewesen sei, kategorisch aus. Entscheidend für die Haftung war erneut die Tatsache, dass der Gutachter die Echtheit des Gemäldes behauptet und dadurch beim Käufer einen Irrtum hervorgerufen hatte, der schließlich zur Ersteigerung des Bildes führte.

Obwohl diese Ausgestaltung der Expertenhaftung den allgemeinen haftungsrechtlichen Grundsätzen widerspricht, ist sie in anderen Entscheidungen bestätigt und verfeinert worden $^{46}$. So finden sich sogar Entscheidungen, in denen zugestanden wird, dass nur die Zerlegung des Werkes, die zwar weder vereinbart noch den Standards der Untersuchung entspreche, erlaubt hätte, die Fälschung festzustellen ${ }^{47}$. Entscheidend sei aber die Behauptung des Gutachters, es handele sich um ein echtes Werk, da diese Erklärung die Haftung gegenüber dem Käufer begründe.

Insgesamt scheint es daher nicht übertrieben, von einer regelrechten "Kataloghaftung" des Gutachters zu sprechen, die als Garantiehaftung weit über die Haftung für eine Sorgfaltspflichtverletzung hinausreicht ${ }^{48}$.

Angesichts des weitreichenden Charakters der Haftungsfolge stellt sich die Frage ihrer Berechtigung.

\section{Die Hauptargumente der richterrechtlichen Garantiehaftung}

In den gerichtlichen Entscheidungen lassen sich zwei Argumente zur Herleitung der Haftung ausmachen: Das erste ist der Hinweis darauf, der Experte habe dem Auktionator bzw. Verkäufer, der in der Hauptsache vom Käufer auf Nichtigkeit des Kaufes

45 Cass. civ. 1re, 3 avril 2007, no 05-12.238, Bull. civ. n 141: „Mais attendu que la cour d'appel, qui a exactement énoncé que, vis-à-vis de la victime de l'erreur, le commissaire-priseur ou l'expert qui affirme l'authenticité d'une cuvre d'art sans assortir son propos de réserves engage sa responsabilité sur cette assertion (...)".

46 Vgl. Cass. civ. Ire, 28 juin 2007, n 05-20.527 (non-publié), weitere Nachweise bei Duret-Robert (Fn. 5) Rn. 143.02.

47 Lequette-de Kervenoaël (Fn. 10) Rn. 525-527, S. 464-466.

48 Diese gilt auch gegenüber dem professionellen Käufer, vgl. Cass. civ. 1 re, 8 déc. $2009, n^{\circ} 08-16.471$, Bull. civ. $n^{\circ} 240$. 


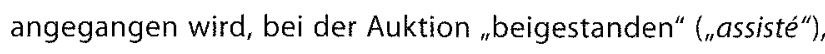
das zweite ist die Nennung - jedenfalls bei der Aufhebung (cassation) der instanzgerichtlichen Entscheidung - eines Dekretes von 1981.

\section{a. Die Funktion des Gutachters im Auktionsverfahren}

Der Hinweis auf den Beistand oder die Unterstützung des Experten bei der Versteigerung greift die Formulierung des Art. L.321-17 C. com. auf ${ }^{49}$, welche auf die Funktion des Experten im Auktionsverfahren, insbesondere auf die Bedeutung, die das Gutachten im Rahmen der Auktion hat ${ }^{50}$, verweist. Aus diesem Grund, dürfte der Hinweis auf den Beistand des Auktionators durch den Experten als ein Argument auf die vertragliche Bindung zwischen Auktionator und Experte zu lesen sein. Der Gutachter haftet also aufgrund seiner Funktion im Auktionsverfahren, die dem Vertrag zwischen Gutachter und Auktionator schon nach der gesetzlichen Formulierung zugrunde liegt. Dieser Betrachtungsweise entspricht, dass der Auktionator für die Auswahl eines geeigneten und kompetenten Experten haftet ${ }^{51}$.

Die so hergestellte Verbindung zwischen Gutachter und Auktionator erhält ihre Brisanz durch das zweite Argument, das die Cour de cassation den Instanzgerichten entgegenhält. Es handelt sich um einen nicht-legislativen Text, nämlich um eine Rechtsverordnung des Premierministers, ein Dekret ${ }^{52}$.

\section{b. Das Dekret Nr. 81-255 vom 3. März 1981}

Grundlage der strengen Kontrolle der Experten durch die Cour de cassation ist das Dekret Nr. 81-255 vom 3. März 1981 über die "Ahndung von Betrug im Rahmen des Handels von Kunstund Sammlergegenständen ${ }^{\mu 53}$. Auf den ersten Blick hat dieses Dekret, das sich der Betrugsbekämpfung, insbesondere der Einhaltung verbindlicher Standards bei der Abfassung von Katalogen, verschrieben hat, nichts mit einer Haftung des Gutachters zu tun ${ }^{54}$. Zwar legt es etwa verbindlich fest, welche Bedeutung bestimmte Formulierungen in der Beschreibung des Werkes haben, das Dekret richtet sich aber ausschließlich an den Auktionator, der sich gegenüber den Käufern nicht durch verhüllende Formulierungen aus der Haftung entziehen soll ${ }^{55}$.

49 Vgl. Duret-Robert (Fn. 16) S. 51.

50 Vgl. Lequette-de Kervenoaël (Fn. 10) Rn. 508, S. 448 f.; DuretRobert (Fn. 5) Rn. 141.32.

51 Einzelheiten bei Lequette-de Kervenoaël (Fn. 10) Rn. 516, S. 455 f.; Duret-Robert (Fn. 5) Rn. 141.32 mwN.

52 In der Sache „Dufy" wurde das Décret nicht genannt. Nach Ansicht der Doktrin hat es gleichwohl die Entscheidung begründet, vgl. z.B. Baillet Bouin (Fn. 43) 2288-2292.

53 Décret $n^{\circ} 81-255$ du 3 mars 1981, sur la répression des fraudes en matière de transactions d'cuvres d'art et d'objets de collection.

54 Vgl. Lequette-de Kervenoaël (Fn. 10) Rn. 501, S. 443; abweichend Duret-Robert (Fn. 5) Rn. 143.24.

55 Vgl. Décret $\mathrm{n}^{\circ} 81-255$, Article 1: „Les vendeurs habituels ou occasionnels d'ouvres d'art ou d'objets de collection ou leurs mandataires, ainsi que les officiers publics ou ministériels et les personnes habilitées procédant à une vente publique aux enchères doivent, si l'acquéreur le
Dass dieses, den Auktionator verpflichtende Dekret für die Haftung des Experten gegenüber dem Auktionator(!) und gegenüber dem Käufer als Dritten herangezogen werden kann ${ }^{56}$, ergibt sich, wenn man die zitierte Rechtsprechung der Assemblée Plénière von 2006 erneut betrachtet: Wenn es zutrifft, dass der Auktionator den Experten "heranzieht", beide also gemeinsam die Versteigerung durchführen, so leuchtet ein, dass der Versteigerer bei der Vergabe des Auftrags über das Gutachten den Experten verpflichtet, sowohl die gesetzlichen als auch die verordnungsrechtichen Vorgaben der Begutachtung einzuhatten. Mit anderen Worten: Da der Versteigerer gegenüber seinen Käufern an die Formulierungen des Dekretes gebunden ist, wird er darauf achten (bzw. kann man den Vertrag entsprechend auslegen), dass auch das Gutachten diese Formulierungen entsprechend verwendet. Äußert mithin ein Experte die Ansicht, das Werk sei einem bestimmten Künstler zuzuschreiben, kann dies aufgrund der vertraglichen Abrede zwischen Experte und Versteigerer keine andere Bedeutung haben als für den Versteigerer im Verhältnis zum Kunden ${ }^{57}$. Wenn aber das Dekret insoweit auch auf das Vertragsverhältnis zwischen Versteigerer und Gutachter ausstrahlt, liegt es in der Konsequenz der Rechtsprechung von 2006, den Gutachter auch im Verhältnis zu Dritten, also im Rahmen der deliktischen Haftung, an seiner vertraglichen, das heißt an der durch das Dekret ausgestalteten Pflicht, zu messen.

Durch diese Auslegung der vertraglichen und deliktischen Pflichten im Lichte der Standards des Dekretes wird das an sich nur den Versteigerer bindende Dekret zu einer Haftungsgrundlage für den Gutachter ${ }^{58}$.

\section{c. Weitergehende Konsequenzen der Berufung auf das Dekret von 1981}

Die Heranziehung des Dekretes als Prüfungsmaßstab erlaubt es der Cour de cassation zudem, die Echtheit eines Gemäldes oder Werkes weitestgehend als Rechtsfrage, nämlich als Auslegung des Dekretes, zu behandeln ${ }^{59}$. Auf diese Weise kann sie die Rechtsprechung der Instanzgerichte, die im Rahmen einer Nichtigkeits- oder einer Schadenersatzklage über die Echtheit bzw. die Zusicherung der Echtheit zu entscheiden haben, weitergehend kontrollieren, denn an sich wäre die Echtheit des Gemäldes als Tatfrage zu qualifizieren ${ }^{60}$. Dass diese Kontrolle nicht

demande, lui délivrer une facture, quittance, bordereau de vente ou extrait du procès-verbal de la vente publique contenant les spécifications qu'ils auront avancées quant à la nature, la composition, l'origine et l'ancienneté de la chose vendue."

56 Einige stützen die Rechtsfortbildung daher auf den Katalog, vgl. Labarthe (Fn. 18) 1779.

57 Vgl. Lequette de Kervenoaël (Fn. 10) Rn. 51.8, S. $457 \mathrm{f}$

58 Vgl. Lequette-de Kervenoael (Fn. 10) Rn. 533-1, S. $474 \mathrm{f.}$

59 Vgl. auch F. Labarthe, Erreur sur la substance: fin de la saga de la table, Boulle', Rec. Dalloz 2012, S. 76, 77 f

60 Beispiele für die Nichtigkeitsklage: Cass. civ. 1re, 3 avril 2002, n 99-16.444, Bull. civ. In 111; Cass. civ. 1re, 27 fév. 2007, n 02-13.420, n० 03-21.179, Bull. civ. I n 90, Cass. civ. 1re, 20 oct. 2011, n 10-25.980, Bull. civ. I n 173. 
immer zu zustimmungswürdigen Ergebnissen führt, zeigt der Fall um die "Fallen-Bilder" des schweizerischen Malers Daniel Spoerri $^{61}$. In dem von der Cour de cassation entschiedenen Fall hatte ein Liebhaber dieser Bilder eines erstanden, das im Katalog nur mit dem Hinweis "Spoerri, tableau-piège" ausgewiesen war. Erst nachträglich erfuhr der Erwerber, dass Spoerri nur die Ausführung überwacht und sein Siegel aufgeklebt hatte, der Hersteller des Bildes aber ein sechsjähriges Kind gewesen war. Er klagte auf Aufhebung des Kaufes und Schadenersatz gegen den Gutachter wegen falscher Herkunftsbezeichnung. Die Cour d'appel wies die Klage zurück, indem sie zunächst darauf abstellte, dass es sich tatsächlich um einen „echten“ Spoerri handle, da ja der Künstler selbst das Werk als eigenes bezeichnet habe. Weiter verwies das Gericht darauf, dass sich der Käufer jederzeit hätte informieren können, wie das Bild zustande gekommen war. Die Cour de cassation hob die Entscheidung auf Sie stellte sich auf den Standpunkt, dass der Gutachter, der das Bild als "von Spoerri" beschreibe, zusichere, dass es tatsächlich vom Künstler selbst hergestellt worden sei. Abweichende Vorgehensweisen bei der Herstellung des Bildes müssten dem Erwerber offengelegt und sowohl im Gutachten als auch im Auktionskatalog angegeben werden. In der Tat bestimmt Artikel 3 des Dekretes von 1981, dass, wenn nicht ein ausdrücklicher Vorbehalt zur Echtheit aufgenommen wird, die Unterzeichnung oder der Stempel des Künstlers die Garantie beinhaltet, dass der Künstler tatsächlich der Urheber des Werkes ist ${ }^{62}$.

Indem die Cour de cassation die Vorgaben des Dekrets über die Würdigung der Cour d'appel stellt, unterstreicht sie ihren Willen, die Echtheit eines Werkes als Rechtsfrage überwachen zu wollen, setzt sich aber in eklatanten Widerspruch zur Definitionsmacht des Künstlers. In der Konsequenz hatte der Gutachter dafür einzustehen, dass sich der Künstler selbst nicht an die Vorgaben des Dekretes gehalten hatte.

Auch die weitere Ausgestaltung der Expertenhaftung durch die Rechtsprechung führt zu einer weitreichenden und nicht immer klar begrenzten Verantwortlichkeit des Gutachters im Verhältnis zum Erwerber des sich später als unecht erweisenden Kunstwerkes.

\section{Die weitere Ausgestaltung der richterrechtlichen Garantiehaftung}

Die Weite der Haftung zeigt sich mit Blick auf die Haftungsausfüllung, die Solidarhaftung von Experte und Auktionator sowie die Verjährung.

\section{a. Haftungsausfüllung}

Hinsichtlich der Haftungsausfüllung gilt sowohl für die Vertrags- als auch für die Deliktshaftung grundsätzlich das Prinzip des vollen Ausgleichs (réparation intégrale) ${ }^{63}$. Dabei wird traditionell der kompensatorische Schaden vom Verzugsschaden unterschieden ${ }^{64}$. Der kompensatorische Ersatz umfasst den materiellen und immateriellen Schaden, wobei bemerkenswert ist, dass Ersatz des immateriellen Schadens (dommage moral) nach Vertrags- wie Deliktsrechts erlangt werden kann ${ }^{65}$, ohne dass dieser auf die Folgen bestimmter Körper- oder Personenschäden begrenzt ist. Aus diesem Grund kann der Käufer eines unechten Werkes geltend machen, er sei - über die finanziellen Einbußen zum Beispiel bei einem Kunstwerk als Geldanlage hinaus - in seiner Erwartung enttäuscht worden, ein echtes Kunstwerk sein Eigen zu nennen, also Stolz und Glück bei diesem Gedanken zu empfinden ("préjudice de l'amateur abusé" oder "sentiment d'avoir été trompé $\left.{ }^{\prime \prime 66}\right)$. Diese, vom Gericht zu schätzende Summe tritt mithin neben den ohnehin geschuldeten Ersatz frustrierter Aufwendungen (Transport- und Lagerungskosten ${ }^{67}$ ), den Ersatz finanzieller Einbußen durch die Fehlinvestition (kein Mehrwert des Bildes nach zehn Jahren ${ }^{68}$ ) und den unter Umständen vom Gutachter zu zahlenden Schadenersatz für den verloren gegangen Kaufpreis ${ }^{69}$.

Somit kann sich der vom Gutachter zu verantwortende Schaden im Ergebnis auf ein Vielfaches des Honorars belaufen; vor allem aber legt diese Rechtsprechung dem Gutachter das Vertragsrisiko zwischen Auktionator und Erwerber auf, indem sie dem Erwerber die Möglichkeit eröffnet, im Rahmen des Schadenersatzes auch den Kaufpreis vom Gutachter zu verlangen, den der Erwerber im Rahmen der Auktion gezahlt hat. Und dies soll sogar dann gelten, wenn der Erwerber nicht einmal versucht hat, die Nichtigkeitsklage wegen Irrtums zu erheben,

$63 \mathrm{Zu}$ Einzelheiten vgl. nur G. Viney/P. Jourdain, Les effets de la responsabilité, in: Ghestin (Fn. 26) 2011, Rn. 57-62, S. 154-181.

64 C. Bloch, in: Le Tourneau (Fn. 26) Rn. 2454; zum Prinzip des immateriellen Schadens vgl. auch Viney/Jourdain (Fn. 26) Rn. 253-270, S. 34-73 mwN.

65 Zur Anerkennung des immateriellen Schadens auch bei Vertragsverletzung vgl. Lequette-de Kervenoaël (Fn. 10) Rn. 492, S. 437 $\mathrm{mwN}$.

66 Vgl. Lequette-de Kervenoaël (Fn. 10) Rn. 549, S. 489

67 Vgl. Lequette-de Kervenoaël (Fn. 10) Rn. 548, S. 486.

68 Es handelt sich mithin um den Ersatz des Schadens, der bei zutreffender Begutachtung hätte vermieden werden können. Zur umstrittenen Dogmatik der perte d'une chance ist hier nicht Stellung zu nehmen. Vgl. dazu Viney/Jourdain (Fn. 26) Rn. 280-284, S. 91-103.

69 Vgl. oben unter (II. 1). 
also Rückerstattung der gezahlten Summe zu verlangen ${ }^{70}$. Dies wird auch mit der Überlegung gerechtfertigt, dass der Experte ansonsten dem Verkäufer auf den Schaden zu haften hätte, den dieser durch das fehlerhafte Gutachten erleidet, wozu auch gehören könne, dass er den Kaufpreis an den Käufer zu erstatten habe ${ }^{71}$.

Zu dieser Betrachtungsweise fügt sich die gesamtschuldnerische Haftung von Gutachter und Auktionator ${ }^{72}$.

\section{b. Gesamtschuldnerische Haftung von Gutachter und Auktionator}

Die in Art. L. 321-30 C. com. statuierte Gesamtschuld (responsabilité in solidum) ist ursprünglich eine Erfindung der Rechtsprechung $^{73}$. Als Zweck der Vorschrift wird auf den Schutz des Gläubigers, das heißt des enttäuschten Käufers, verwiesen, der sich bei Zahlungsunfähigkeit des Auktionators oder des Gutachters jeweils an den anderen halten könne ${ }^{74}$. Da mittlerweile eine Versicherungspflicht für beide Verpflichteten statuiert worden ist, dürfte diese Schutzwirkung selten praktisch relevant werden $^{75}$, sodass die Aufteilung der Haftung im Innenverhältnis zwischen Gutachter und Auktionator in den Mittelpunkt rückt. Die Rechtsprechung stellt hier regelmäßig darauf $a b$, dass der Gutachter den Auftrag habe, dem Auktionator durch seine Expertise eine Grundlage für die Auktion zu bieten ${ }^{76}$. Die Letztverantwortlichkeit wird daher meist überwiegend dem Gutachter zugewiesen ${ }^{77}$, wobei sich prozessual die Möglichkeit bietet ("appel en garantie"), die anteilsmäßige Verteilung des Schadens schon im Rahmen der Schadenersatzklage des Käufers gegen Versteigerer und Gutachter festzustellen ${ }^{78}$. Neben der alleinigen Verantwortlichkeit des Gutachters sind auch anteilige Haftungszuweisungen zu beobachten, so etwa die Haftung des Gutachters zu zwei Dritteln, des Versteigerers zu einem Drittel, etwa weil der Versteigerer den Fehler des Gutachters mitverursacht habe ${ }^{79}$. Diese Haftungsverteilung zeigt erneut, dass sich der Auktionator durch die Beiziehung des Gutachters teilweise aus seiner eigenen Haftung befreien kann.

70 Vgl. Cass. civ. Ire, 23 avril 1969, Bull civ. I n 148 , D. 1969, jur 493. Weitere Entscheidungen der Instanzgerichte bei DuretRobert (Fn. 5) Rn. 144.41 f.

71 Vgl. Duret-Robert (Fn. 5) Rn. 144.67.

72 Haftungsbegrenzungsklauseln sind unwirksam, vgl. Art. L. 321-17 Abs. 2 C. com.: "Les clauses qui visent à écarter ou à limiter la responsabilité (des opérateurs de ventes et des experts) sont interdi. tes et réputées non écrites."

73 Baillet Bouin (Fn. 43) 2292; Duret-Robert (Fn. 16) S. 51; zuletzt ders. (Fn. 5) Rn. 141.41-141.42.

74. Duret-Robert (Fn. 16) S. $51 \mathrm{f}$.

75 Duret-Robert (Fn. 16) S. 52.

76 Vgl. Baillet Bouin (Fn. 43) 2291

77 Ein Überblick über die Stadien der Rechtsprechung bei DuretRobert (Fn. 5) Rn. 141.50-141.53.

78 Vgl. Lequette-de Kervenoaël (Fn. 10) Rn. 558, S. 495.

79 Lequette-de Kervenoael (Fn. 10) Rn. 559, S. 496 f.; Baillet Bouin (Fn. 43) 2292. Vgl. auch C.A. Paris, 1re ch. sect. A, 24 févr. 2009, RG n 07-05369, TGI Paris, 5e ch. 1re sect., 29 sept. 2009, RG n 08-10650.
Dagegen hat die Rechtsprechung die Expertenhaftung auch zeitlich, das heißt mit Blick auf die Verjährungsregeln, gegenüber dem allgemeinen Haftungsregime verschärft.

\section{c. Zur Verjährung der Haftung}

Nach der 2011 bestätigten Fassung gilt für die Haftung von Gutachtern und Auktionaren bei Versteigerungskäufen die in Art. L. 321-17 Abs. 3 C. com. bestimmte Verjährungsfrist von fünf Jahren nach dem Zuschlag. Die Cour de cassation weigert sich aber, diese Vorschrift auf die Haftung des Gutachters für seine Expertise anzuwenden und rekurriert auf die Regelverjährung, die seit 2008 ebenfalls fünf Jahre beträgt, aber mit dem Tage zu laufen beginnt, an dem der Rechtsinhaber die Tatsachen gekannt hat oder hätte kennen müssen, die es ihm erlaubten, sein Recht auszuüben (Art. 2224 C. civ.). Stellt sich die Unechtheit des Bildes erst nach einigen Jahren heraus, bedeutet dies de facto eine Verlängerung des Haftungszeitrums, da der Käufer nach dieser Rechtsprechung seine Haftungsklage auch noch später als fünf Jahre nach dem Zuschlag erheben kann. Zur Begründung wird darauf verwiesen, die in Art. L. 321-17 Abs. $3 \mathrm{C}$. com. verwendete Formulierung "actions en responsabilité civile (Haftungsklagen) betreffe nicht die Haftung für Gutachten, da diese nicht auf einem Fehiverhalten bei der Versteigerung selbst beruhe, sondern auf der fehlerhaften Fassung des Versteigerungskatalogs. ${ }^{80}$ Erneut dient das Abstellen auf die Katalogangaben mithin dazu, eine gesetzliche Regelung, die zu einer Haftungsbeschränkung führen würde, auszuschalten. Seit der Verjährungsreform im Jahre 2008 wird diese Erweiterung auch damit gerechtfertigt, dass der Verjährungsbeginn regelmäßig an die Erkenntnis des Schadens geknüpft sei, weshalb Art. L. 321-17 Abs. 3 C. com., der auf den Zuschlag abstelle, als Ausnahmeregelung restriktiv ausgelegt werden müsse. ${ }^{81}$

\section{Fazit}

Fasst man die Verantwortlichkeit der Beteiligten im Kunstmarkt nach französischem Recht zusammen, so kann man sich des Eindrucks nicht erwehren, dass der Schutz des Erwerbers auf Kosten des Gutachters erkauft worden ist: Da der Gutachter am Ende der Kette von Einlieferer, Versteigerer und Käufer steht und sein Gutachten Grundlage der hier getätigten Geschäfte bildet, ist er derjenige, der für die Fehleinschätzung der Authentizität des Werkes - und sei diese Fehleinschätzung auch unverschuldet - einzustehen hat.

80 Vgl. C.A. Paris, 1re ch. sect. A, 14 juin 2011, n 09/24375: „(..) considérant (...) que la prescription qu'il invoque ne peut s'appliquer au présent litige, le texte auquel il se réfère concernant les actions en responsabilité civile engagées à l'occasion des prisées et des ventes volontaires (...), ce qui n'est pas le cas de l'action en dommages et intérêts de Mme X..., laquelle ne remet pas en cause la vente elle-même, mais recherche la responsabilité du commissaire-priseur et de l'expert en raison de fautes commises dans la rédaction du catalogue".

81 Vgl. Duret-Robert (Fn. 5) Rn. 145.11-145.22. Ähnlich Cass. civ. 1re, 17 nov. 2011, $\mathrm{n}^{\circ} 10-26.536$ (non publié) zur Verjährungsfrist aus Art. L. 110-4, I, C. com. 
Grundlage dieser Haftung ist eine schöpferische Rechtsprechung (jurisprudence créatrice), die sich dem Anliegen verschrieben hat, einen möglichst betrugsfreien Kunstmarkt zu schaffen. ${ }^{82}$ Dabei setzt sich die französische Rechtsprechung über die gültigen Regeln der Vertrags- bzw. der Deliktshaftung hinweg und schafft ein Sonderregime der Gutachterhaftung im Kunstmarkt. Der in dieser Schöpfung liegende Widerspruch zu allgemeinen haftungsrechtlichen Prinzipien wird auch von der französischen Doktrin hervorgehoben. ${ }^{83}$ In der Tat ist es frag- würdig, die Unsicherheiten, die mit dem Erwerb im Kunsthandel verbunden sind, vorrangig den Gutachtern aufzubürden. Vor allem aber erweist sich die lediglich auf ein Dekret gestützte Rechtsprechung zur Gutachterhaftung als konträr zum gesetzgeberischen Willen, den Kunstmarkt und insbesondere den Auktionshandel zu liberalisieren und sich - zur Stärkung der Attraktivität des französischen Standortes - von einem etatistischen Verständnis des Auktionswesens zu verabschieden.

82 Duret-Robert (Fn. 16) S. 51: "La position de la Cour de cassation peut paraître sévère. Il semble, en effet, difficile d'imposer à un professionnel, dans un domaine aussi aléatoire que celui de lexpertise, une quasi-obligation de résultat."

83 Duret-Robert (Fn. 16) S. 58. Hier dürfte der Versicherungsge danke von Bedeutung sein, vgl. Art. 321-30 Abs. 1 C. com: "Tout expert intervenant à titre onéreux à l'occasion d'une vente de meubles aux enchères publiques est tenu de contracter une assurance garantissant sa responsabilité professionnelle."

\section{Keine Justiz ist unfehlbar!}

Sabine Weber*

Der französische Ex-Justizminister Robert Badinter schreibt ein Opernlibretto und macht sein Lebensthema zu einem klingenden Vermächtnis: Den Kampfgegen Todesstrafe und Ungerechtigkeit als Folge von Rechtssystemen, wozu er einen inhumanen Justizvollzug zählt. Das Gefängnisdrama „Claude" hat Thierry Escaich, Organist, Komponist und Kompositionsprofessor in Paris, mit über weite Strecken bedrohlich pulsierenden Klängen unterfüttert. Regisseur Oliver Py hat das Bühnenbild von Pierre-André Weitz mit szenischem Furor belebt. Am 27. April 2013 war die Uraufführung an der Oper in Lyon. Im Rahmen des Festivals „Justice/Injustice“, das um eben solche Fragen gekreist ist.

- Wenn irgendwo auf dieser Welt gegen ein Todesurteil protestiert wird, wie zuletzt im Fall des in den USA im Bundesstaat Georgia hingerichteten Troy Davis, ist immer auch seine Stimme in den Medien präsent. Seit 1972 kämpft der 1928 geborene Jurist Robert Badinter leidenschaftlich gegen die Todesstrafe. In Büchern hat er diesen Kampf dokumentiert. In "L'Éxecution" von 1973 verarbeitet er das Trauma einer verlorenen Strafvertei-

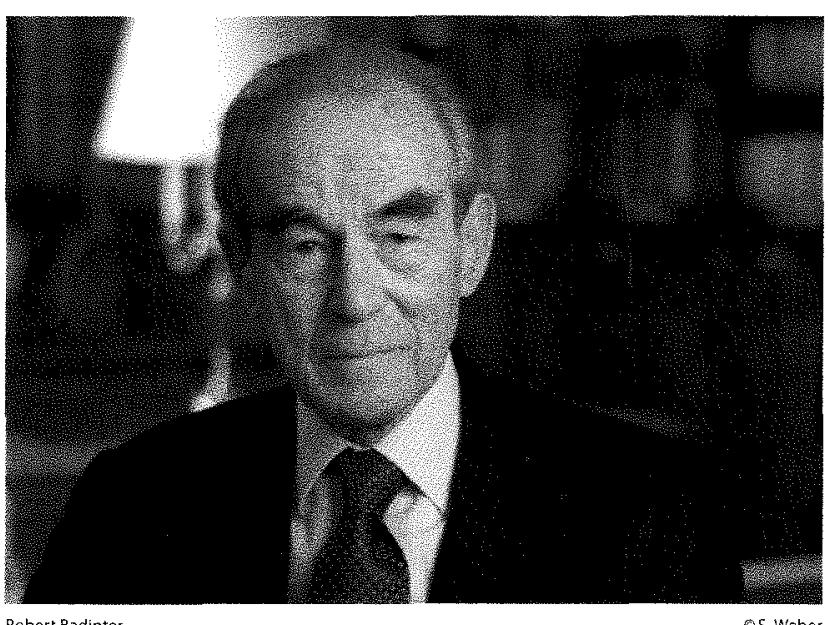

digung vor dem Geschworenengericht in Troyes. Seinen Mandanten kann er nicht vor der Guillotine retten, auch, weil der damalige Präsident Georges Pompidou das Gnadengesuch ablehnt. Von François Mitterrand dann zum Justizminister berufen, initiiert Robert Badinter 1981 die Debatte vor der Assemblée Nationale. Nach der Abstimmung gehört die Justiz, die tötet, der französischen Vergangenheit an. Dennoch: die Zeitung "Le Figaro" hat eine Meinungsumfrage durchgeführt, die sie am Morgen der Abstimmung veröffentlicht. 62 Prozent der Franzosen hätten für die Todesstrafe gestimmt, so Badinter, nachzulesen in seinem 2000 veröffentlichten Buch "L'Abolition". Popularität hat Badinter durch diesen Akt nicht gewonnen. Oder „Monsieur Abolition" wie ihn seine Gegner nennen. Und immer, wenn ein fürchterliches Verbrechen passiere, flamme die Diskussion wieder auf. Also hört er nicht auf, für seine Überzeugung zu kämpfen und hat ein Opernlibretto verfasst, das von einem zum Tode Verurteilten handelt. Von einem Sträfling, der sich gegen die Ungerechtigkeit seiner Verurteilung und gegen den brutalen Justizvollzug auflehnt.

* Sabine Weber ist Kulturjournalistin in Köln 